\title{
Brans-Dicke brane cosmology
}

\author{
Luís E. Mendes ${ }^{\dagger}$ and Anupam Mazumdar* \\ ${ }^{\dagger}$ Astronomy Centre, University of Sussex, Falmer, BN1 9QH, U. K \\ ${ }^{*}$ Astrophysics Group, Blackett Laboratory, Imperial College, London SW7 2BW, U. K
}

(October 25, 2018)

\begin{abstract}
A five dimensional brane cosmology with non-minimally coupled scalar field to gravity is considered in the Jordan frame. We derive the effective four dimensional field equations on a $3+1$ dimensional brane where the fifth dimension is assumed to have an orbifold symmetry. We show that energy conservation still holds for matter on the brane in spite of the existence of a scalar field (the Brans-Dicke field) in the bulk. Finally, we discuss some cosmological consequences of this setup.
\end{abstract}

\section{INTRODUCTION}

Recently the possibility that the observable Universe is a brane-world [1] embedded in a higher dimensional spacetime has generated a great deal of interest. Such a claim is motivated by the strongly coupled sector of $E_{8} \times E_{8}$ heterotic string theory which can be described by a field theory living in 11 dimensional space-time [2]. The 11 dimensional world is comprised of two 10 dimensional hypersurfaces embedded on the fixed points of an orbifold and the matter fields are assumed to be confined to these hypersurfaces which in this scenario are known to be 9 branes. After compactifying the 11 dimensional theory on a Calabi-Yau three fold, one obtains an effective 5 dimensional theory [3] which has a structure of two 3 branes situated on the orbifold boundaries. The theory allows $N=1$ supergravity with gauge and chiral multiplets on the two 3 branes. Motivated by the above setup there has been an attempt to understand the case where the bulk is 5 dimensional anti de-Sitter space. It was shown that gravity could also be localized in this scenario [ـ. These authors demonstrated that in a background of a special non-factorizable geometry an exponential warp factor multiplies the Poincaré invariant $3+1$ dimensions in the metric. The model consists of two 3 branes situated rigidly along the 5 th dimension compactified on a $S^{1} / Z_{2}$ orbifold symmetry. In order to realize this simple scenario the two branes must have opposite tensions.

It was realized that the brane-world construction could modify the early Universe considerably and, furthermore, the two branes with opposite tensions should behave differently [5]. The most important observation was the departure of the evolution equation on the brane from the standard four dimensional evolution equation when no branes are present [6]. The presence of branes and the requirement that the fields should be localized lead to a non-conventional cosmology which requires a more exhaustive study. Some attention has been devoted to the effective gravity induced on the brane [7] and a great emphasis was placed on inflationary cosmology [8] and, more recently, post-inflationary brane cosmology has been considered in Ref. [9]. Apart from inflationary and post-inflationary brane cosmology, there has been a great deal of interest in the four dimensional cosmological constant problem [10 12]. These authors have considered a five dimensional action with a scalar field non-minimally coupled to the five dimensional gravity and to the four dimensional brane tension. There has also been some discussion on the localization of gravity in this setup [13]. In this paper we would like to extend the calculation made in Ref. [6] to include the dynamics of a scalar field which lives on the brane and the bulk. We then study the effective field equations on the $3+1$ dimensional brane locally and globally in presence of the other brane which is assumed to be rigidly located on the orbifold symmetry along the 5th dimension. Finally, we discuss the pertaining cosmology.

\section{BRANS-DICKE BRANE MODEL IN 5 DIMENSIONS}

In this article we restrict ourselves to one extra space-like dimension with a scalar field non-minimally coupled to gravity in 5 dimensions. We analyze the physics in the Jordan frame where the interpretation of the results is straightforward, as we shall see in the next sections. We believe that such a scalar field in the five dimensional theory can be a dilaton which is purely an outcome of dimensional reduction from some higher dimensional theory to 5 dimensional space-time 円. We assume the following action for the rest of our discussion

\footnotetext{
${ }^{1}$ This might not be the case if gravity had to be localized in a particular brane (see Ref. [14]).
} 


$$
S_{5}=-\frac{1}{2 \kappa_{(5)}^{2}} \int d^{5} x \sqrt{-\tilde{g}}\left(\phi \tilde{R}-\frac{\omega}{\phi} \partial_{\mathrm{A}} \phi \partial^{\mathrm{A}} \phi\right)+\int d^{5} x \sqrt{-\tilde{g}} \mathcal{L}_{\mathrm{m}}
$$

where $\tilde{R}$ is the Ricci scalar associated with the 5 -dimensional space-time metric $\tilde{g}_{\mathrm{AB}}, \phi$ is a scalar field which we will call Brans-Dicke (BD) field, $\omega$ is a dimensionless coupling constant which determines the coupling between gravity and the BD scalar field and finally, $\mathcal{L}_{\mathrm{m}}$ represents the Lagrangian for the matter fields. Latin indices denote 5 dimensional components $(\mathrm{A}, \mathrm{B}=0, \ldots, 5)$ and for our convenience we choose $\kappa_{5}^{2}=1$. The variational derivative of the action Eq. (11) with respect to $g_{\mathrm{AB}}$ and $\phi$ yields the field equations 15

$$
\begin{aligned}
\tilde{R}_{\mathrm{AB}}-\frac{1}{2} \tilde{g}_{\mathrm{AB}} \tilde{R} & =\frac{\omega}{\phi^{2}}\left[\phi_{; \mathrm{A}} \phi_{; \mathrm{B}}-\frac{1}{2} \tilde{g}_{\mathrm{AB}} \phi_{; \mathrm{C}} \phi^{; \mathrm{C}}\right]+\frac{1}{\phi}\left[\phi_{; \mathrm{AB}}-\tilde{g}_{\mathrm{AB}} \phi_{; \mathrm{C}} ; \mathrm{C}\right]+\frac{\tilde{T}_{\mathrm{AB}}}{\phi}, \\
\square \phi & =\frac{\tilde{T}}{3 \omega+4},
\end{aligned}
$$

where $\tilde{T}=\tilde{T}^{\mathrm{C}}{ }_{\mathrm{C}}$ is the trace of the energy-momentum tensor of the matter content of 5 dimensional space-time. Notice the $3 \omega+4$ denominator in the right hand side of the BD field equation instead of the familiar $2 \omega+3$ in the 4 -dimensional case 16]. This is determined by requiring the validity of the equivalence principle in our setup (see Weinberg's book 15] for a discussion of this topic in the context of 4-dimensional Brans-Dicke theory).

Before we discuss the energy-momentum tensor, let us define the five dimensional metric which has the following form

$$
d s^{2}=\tilde{g}_{\mathrm{AB}} d x^{\mathrm{A}} d x^{\mathrm{B}}=g_{\mu \nu}\left(x^{\mu}, y\right) d x^{\mu} d x^{\nu}+b^{2}\left(x^{\mu}, y\right) d y^{2},
$$

where $\mu, \nu=0, \ldots, 3$, and $y$ is the coordinate associated with the fifth dimension which is assumed to be compact with a range $-1 / 2 \leq y \leq 1 / 2$. We also assume an orbifold symmetry along the fifth direction $y \rightarrow-y$. As we shall see in the coming sections, this will help us to simplify our calculation. Since the opposite points along the fifth dimension are identified, we will only be interested in the interval $0 \leq y \leq 1 / 2$, which is the spacing between the two branes situated at $y=0$ and $y=1 / 2$. Next we define the energy-momentum tensor

$$
\tilde{T^{\mathrm{A}}}{ }_{\mathrm{B}}=\left.T^{\mathrm{A}}{ }_{\mathrm{B}}\right|_{\text {bulk }}+\left.T^{\mathrm{A}}{ }_{\mathrm{B}}\right|_{\text {brane }},
$$

where the subscripts brane and bulk suggest the corresponding energy-momentum tensors. For simplicity we assume the bulk is devoid of matter other than the $\mathrm{BD}$ scalar field. The brane matter fields are stuck at $y=0$ and $y=1 / 2$ with the following energy momentum tensor

$$
\begin{aligned}
\left.T_{\mathrm{B}}^{\mathrm{A}}\right|_{\text {brane }} & =\frac{\delta(y)}{b} \operatorname{diag}(-\rho, p, p, p, 0), \\
\left.T_{\mathrm{B}}^{\mathrm{A}}\right|_{\text {brane* }} & =\frac{\delta(y-1 / 2)}{b} \operatorname{diag}\left(-\rho_{*}, p_{*}, p_{*}, p_{*}, 0\right),
\end{aligned}
$$

where the subscript ' $*$ ' corresponds to the matter on the brane at $y=1 / 2$. While writing the above expressions we have taken the infinitely thin brane limit.

\section{EQUATIONS OF MOTION}

Since we are interested in exploring the flat cosmology, we consider a 5 dimensional flat metric ansatz of the following form

$$
d s^{2}=-n^{2}(\tau, y) d \tau^{2}+a^{2}(\tau, y) \delta_{i j} d x^{i} d x^{j}+b^{2}(\tau, y) d y^{2},
$$

where $i, j=1,2,3$. With this metric ansatz we are now able to write the equations of motion. The $(0,0)$ component reads

$$
\begin{aligned}
3\left[\frac{\dot{a}}{a}\left(\frac{\dot{a}}{a}+\frac{\dot{b}}{b}\right)-\frac{n^{2}}{b^{2}}\left(\frac{a^{\prime \prime}}{a}+\frac{a^{\prime}}{a}\left(\frac{a^{\prime}}{a}-\frac{b^{\prime}}{b}\right)\right)\right]= \\
\quad-\frac{\dot{\phi}}{\phi}\left(3 \frac{\dot{a}}{a}+\frac{\dot{b}}{b}-\frac{\omega}{2} \frac{\dot{\phi}}{\phi}\right)+\left(\frac{n}{b}\right)^{2}\left[\frac{\phi^{\prime \prime}}{\phi}+\frac{\phi^{\prime}}{\phi}\left(3 \frac{a^{\prime}}{a}-\frac{b^{\prime}}{b}+\frac{\omega}{2} \frac{\phi^{\prime}}{\phi}\right)\right]+\frac{\tilde{T}_{00}}{\phi}
\end{aligned}
$$


the $(i, j)$ component is given by

$$
\begin{aligned}
\left\{-2 \frac{\ddot{a}}{a}-\frac{\ddot{b}}{b}+\right. & {\left.\left[\frac{\dot{a}}{a}\left(-\frac{\dot{a}}{a}+2 \frac{\dot{n}}{n}\right)+\frac{\dot{b}}{b}\left(-2 \frac{\dot{a}}{a}+\frac{\dot{n}}{n}\right)\right]+\left(\frac{n}{b}\right)^{2}\left[2 \frac{a^{\prime \prime}}{a}+\frac{n^{\prime \prime}}{n}+\frac{a^{\prime}}{a}\left(\frac{a^{\prime}}{a}+2 \frac{n^{\prime}}{n}\right)-\frac{b^{\prime}}{b}\left(\frac{n^{\prime}}{n}+2 \frac{a^{\prime}}{a}\right)\right]\right\} \delta_{i j}=} \\
& \left\{\frac{\ddot{\phi}}{\phi}+\frac{\dot{\phi}}{\phi}\left(2 \frac{\dot{a}}{a}+\frac{\dot{b}}{b}-\frac{\dot{n}}{n}+\frac{\omega}{2} \frac{\dot{\phi}}{\phi}\right)-\left(\frac{n}{b}\right)^{2}\left[\frac{\phi^{\prime \prime}}{\phi}+\frac{\phi^{\prime}}{\phi}\left(2 \frac{a^{\prime}}{a} \frac{b^{\prime}}{b}+\frac{n^{\prime}}{n}+\frac{\omega}{2} \frac{\phi^{\prime}}{\phi}\right)\right]\right\} \delta_{i j}+\left(\frac{n}{a}\right)^{2} \frac{\tilde{T}_{i j}}{\phi},
\end{aligned}
$$

the $(0,5)$ component takes the form

$$
3\left(\frac{\dot{a}}{a} \frac{n^{\prime}}{n}+\frac{\dot{b}}{b} \frac{a^{\prime}}{a}-\frac{\dot{a}^{\prime}}{a}\right)=\frac{\dot{\phi}^{\prime}}{\phi}-\frac{\dot{\phi}}{\phi}\left(\frac{n^{\prime}}{n}-\omega \frac{\phi^{\prime}}{\phi}\right)-\frac{\dot{b}}{b} \frac{\phi^{\prime}}{\phi},
$$

and, finally, for the $(5,5)$ component one has

$$
\begin{aligned}
3\left[-\left(\frac{\ddot{a}}{a}+\frac{\dot{a}}{a}\left(\frac{\dot{a}}{a}-\frac{\dot{n}}{n}\right)\right)+\left(\frac{n}{b}\right)^{2}\left(\frac{a^{\prime}}{a}\left(\frac{a^{\prime}}{a}+\frac{n^{\prime}}{n}\right)\right)\right]= \\
\frac{\ddot{\phi}}{\phi}+\frac{\dot{\phi}}{\phi}\left(3 \frac{\dot{a}}{a}-\frac{\dot{n}}{n}+\frac{\omega}{2} \frac{\dot{\phi}}{\phi}\right)-\left(\frac{n}{b}\right)^{2} \frac{\phi^{\prime}}{\phi}\left(3 \frac{a^{\prime}}{a}+\frac{n^{\prime}}{n}-\frac{\omega}{2} \frac{\phi^{\prime}}{\phi}\right)+\left(\frac{n}{b}\right)^{2} \frac{\tilde{T}_{55}}{\phi} .
\end{aligned}
$$

The equation of motion for the BD field reads

$$
\ddot{\phi}+\dot{\phi}\left(3 \frac{\dot{a}}{a}+\frac{\dot{b}}{b}-\frac{\dot{n}}{n}\right)-\left(\frac{n}{b}\right)^{2}\left[\phi^{\prime \prime}+\phi^{\prime}\left(3 \frac{a^{\prime}}{a}-\frac{b^{\prime}}{b}+\frac{n^{\prime}}{n}\right)\right]=-n^{2} \frac{\tilde{T}}{3 \omega+4}
$$

where the dot corresponds to the time derivative with respect to $\tau$ and the prime corresponds to derivatives with respect to $y$. We make the assumption that the metric and the BD field are continuous across the branes localized at $y=0$ and $y=1 / 2$. However, their derivatives can be discontinuous at the brane positions in the $y$ direction. This suggests the second derivatives of the scale factor and the BD field will have a Dirac delta function associated with the positions of the branes. Since the matter is localized on the branes it will introduce a delta function in the Einstein equations which will be matched by the distributional part of the second derivatives of the scale factor and BD field. For instance at $y=0$, we have

$$
f^{\prime \prime}=\widehat{f^{\prime \prime}}+\left[f^{\prime}\right] \delta(y)
$$

where the hat marks the non-distributional part of the second derivative of the quantity. The part associated with a delta function, $\left[f^{\prime}\right]$, is a jump in the derivative of $f$. Here $f$ could be any of the three quantities $a, n$ or $\phi$. The jump in $f$ at $y=0$ can be written as

$$
\left[f^{\prime}\right]=f^{\prime}\left(0^{+}\right)-f^{\prime}\left(0^{-}\right)
$$

and the mean value of the function $f$ at $y=0$ is defined by

$$
\sharp f \sharp=\frac{f\left(0^{+}\right)+f\left(0^{-}\right)}{2} .
$$

After substituting Eq. (14) in the Einstein equations it is possible to find out the jump conditions for $a$ and $n$ by matching the Dirac delta functions appearing in the left-hand side of the Einstein equations to the ones coming from the energy-momentum tensor Eqs. (57). For the BD field one has to use Eq. (13) to evaluate the jump condition. We get the following results for the jump conditions

$$
\begin{aligned}
& \frac{\left[a^{\prime}\right]_{0}}{a_{0} b_{0}}=-\frac{1}{(3 \omega+4) \phi_{0}}(p+(\omega+1) \rho), \\
& \frac{\left[n^{\prime}\right]_{0}}{n_{0} b_{0}}=\frac{1}{(3 \omega+4) \phi_{0}}(3(\omega+1) p+(2 \omega+3) \rho), \\
& \frac{\left[\phi^{\prime}\right]_{0}}{\phi_{0} b_{0}}=\frac{1}{(3 \omega+4) \phi_{0}}(3 p-\rho)
\end{aligned}
$$


where the subscript ' 0 ' stands for the brane at $y=0$. Similar conditions hold for the brane fixed at $y=1 / 2$ by replacing ' 0 ' by ' $1 / 2$ ' and $\rho$ and $p$ by $\rho_{*}$ and $p_{*}$. The first two conditions, Eqs. (17) and (18), are equivalent to Israel's junction conditions in general relativity [17] (see Ref. [6] for a discussion of its application in the context of brane world). It is important to note that the above jump conditions at $y=0$ depend on the energy density and the pressure component of the brane world. Remarkably for the radiation dominated phase on the brane, $\rho=3 p$, the jump condition for $\phi$ vanishes suggesting that the BD field takes a constant value on the bulk and the brane. This has important consequences which we shall discuss later on.

Taking the jump of the $(0,5)$ component of the Einstein equation and substituting Eqs. (17, 18 ) we get the continuity equation for the matter on the brane

$$
\dot{\rho}+3(\rho+p) \frac{\dot{a}}{a}=0
$$

The fact that the energy content of the brane is still conserved in this scenario seems to be at odds with recent results obtained independently by several authors who conclude that the presence of a dilaton in the bulk will lead to a nontrivial coupling with the matter on the brane which from the point of view of an observer living on the brane would be seen as matter leaking from the brane 12,22, 24. The reason why our situation is different is because the coupling between the BD field and ordinary matter on the brane was chosen in order to satisfy the equivalence principle, as was pointed out above. Had we chosen the coupling in (13) to be the usual 4-dimensional value $(2 \omega+3)^{-1}$ we would end up with a situation where the conservation equation (20) would not hold and energy could leak from the brane.

Taking the mean value (in the sense of Eq. (16)) of the $(5,5)$ component of Einstein's equations we can now obtain a Friedmann type equation on the brane by following a very similar procedure to the one introduced in [6]. Using the fact that due to the orbifold symmetry $y \leftrightarrow-y, \sharp f^{\prime} \sharp=0$ (for $f$ any of the quantities $a, n$ or $\phi$ ), we can discard all the terms involving mean values in the average of the $(5,5)$ component of the Einstein equations. The equation so obtained will still involve a term containing $\ddot{\phi}$, but we can use the mean value of the BD field equation (13) to write this in terms of $a, b$ and $n$, and their derivatives. After a tedious calculation we obtain the Friedmann type equation on the brane

$$
\frac{\ddot{a}_{0}}{a_{0}}+\left(\frac{\dot{a}_{0}}{a_{0}}\right)^{2}+\frac{\omega}{6}\left(\frac{\dot{\phi}_{0}}{\phi_{0}}\right)^{2}=-\frac{1}{24(3 \omega+4) \phi_{0}^{2}}\left[(2 \omega+3) \rho^{2}+6(\omega+1) p \rho+3 p^{2}+\frac{2 \omega(3 p-\rho)^{2}}{3 \omega+4}\right] .
$$

While deriving the above equation we have assumed the extra dimension to be static, $b=b_{0}$, from the point of view of the brane observer and we have also fixed time in such a way that $n_{0}=1$. This corresponds to the usual choice of time in conventional cosmology. It is interesting to note that by taking the limit $\omega \rightarrow \infty$ in the right-hand side of Eq. (21), we obtain exactly the same expression as in general relativity (compare with Eq. (20) in Ref. [6]). However, the above expression differs from the corresponding equation of motion without the presence of branes (see Ref. [16]), namely due to the presence of quadratic terms in $\rho$ and $p$ and the absence of linear terms in the energy density and pressure in (21).

From the mean value of the BD field equation we obtain an equation of motion for $\phi$ on the brane

$$
\ddot{\phi}_{0}+3 \frac{\dot{a}_{0}}{a_{0}} \dot{\phi}_{0}=\frac{\omega(\rho-3 p)^{2}}{4(3 \omega+4)^{2} \phi_{0}} .
$$

Note that in order to obtain Eq. (22) we also have to assume the non-distributional part of $\phi^{\prime \prime}$ vanishes, otherwise, a term involving $\widehat{\phi^{\prime \prime}}$ will appear in the BD field equation. As we shall see in the next Section it is possible to obtain cosmologically interesting solutions which verify this condition. Together with the conservation equation (20), Eqs. (21) and (22) determine the cosmology of the brane at $y=0$. Comparing equation (22) with the usual BD field equation in 4 dimensional BD theory with no branes we note there is an extra power of $\rho-3 p$ and an extra factor of $\phi_{0}^{-1}$ on the right hand side of the $\mathrm{BD}$ field equation in the brane model.

\section{TOPOLOGICAL CONSTRAINTS}

As in the general relativity case, in our setup matter in one brane is also constrained by matter on the second brane (see Ref. [6] for a detailed discussion of this point). In order to get the constraints, we proceed as in Ref. [6]. We will use the following ansatz for the solution

$$
a=a_{0}(t)+\left(\frac{|y|}{2}-\frac{y^{2}}{2}\right)\left[a^{\prime}\right]_{0}-\frac{y^{2}}{2}\left[a^{\prime}\right]_{1 / 2}
$$


where the subscripts ' 0 ' and ' $1 / 2$ ' denote the branes at $y=0$ and $y=1 / 2$ with a similar expression for $n$. As to the $\mathrm{BD}$ field $\phi$, we assume it has the following form

$$
\phi=\phi_{0}(t)+\frac{\left[\phi^{\prime}\right]_{0}}{2}|y| .
$$

Notice that the term quadratic in $y$ which is present in $a$ and $n$ is absent from $\phi$. This difference will be analyzed below. Since $n_{0}(t)$ is a completely arbitrary function which can be fixed by a choice of time coordinate we fix $n_{0}(t)=1$ in order to get the conventional definition of time. For $b$ we use

$$
b=b_{0},
$$

where $b_{0}$ is a constant. This ansatz corresponds to stabilizing the fifth dimension. This is by no means a trivial assumption but for simplicity we assume the fifth dimension to be static. Using the above ansatz for the static extra dimension the $(0,0)$ component of the Einstein equations for the brane situated at $y=0$ yields

$$
\left(\frac{\dot{a}_{0}}{a_{0}}\right)^{2}+\frac{\dot{\phi}_{0}}{\phi_{0}}\left(\frac{\dot{a}_{0}}{a_{0}}-\frac{\omega}{6} \frac{\dot{\phi}_{0}}{\phi_{0}}\right)=\frac{1}{b_{0}^{2}}\left[\frac{1}{4}\left(\frac{\left[a^{\prime}\right]_{0}}{a_{0}}\right)^{2}+\frac{\omega}{24}\left(\frac{\left[\phi^{\prime}\right]_{0}}{\phi_{0}}\right)^{2}+\frac{1}{4} \frac{\left[a^{\prime}\right]_{0}}{a_{0}} \frac{\left[\phi^{\prime}\right]_{0}}{\phi_{0}}-\frac{\left[a^{\prime}\right]_{1 / 2}}{a_{0}}-\frac{\left[a^{\prime}\right]_{0}}{a_{0}}\right] .
$$

In order to be consistent with Eq. (21) the right-hand side of this equation must be quadratic in the energy density. Noticing that the jump conditions for $a$ and $n$ are linear in the energy density, this means that the right-hand side of Eq. (26) must contain only terms quadratic in the jump for the various quantities involved. Terms linear in the jump must therefore vanish yielding the condition

$$
\left[a^{\prime}\right]_{0}=-\left[a^{\prime}\right]_{1 / 2} .
$$

Following a similar procedure with the $(i, j)$ components of the Einstein equations, with the help of the previous constraint Eq. (27) we obtain our last constraint

$$
\left[n^{\prime}\right]_{0}=-\left[n^{\prime}\right]_{1 / 2} .
$$

These constraints have the same form in terms of the jumps in $a$ and $n$ as in the general relativity case. However, using the jump conditions Eqs. (17) and (18) they result in different relations between the energy density and pressure in both branes than the ones found in Ref. [6].

Assuming the matter on the branes obeys an equation of state $p=\epsilon \rho$ for the brane at $y=0$ and $p_{*}=\epsilon_{*} \rho_{*}$ for the brane at $y=1 / 2$, Eqs. (27) and (28) read

$$
[\epsilon+(\omega+1)] \rho \frac{a_{0}}{\phi_{0}}=-\left[\epsilon_{*}+(\omega+1)\right] \rho_{*} \frac{a_{1 / 2}}{\phi_{1 / 2}},
$$

and,

$$
[3(\omega+1) \epsilon+(2 \omega+3)] \rho \frac{n_{0}}{\phi_{0}}=-\left[3(\omega+1) \epsilon_{*}+(2 \omega+3)\right] \rho_{*} \frac{n_{1 / 2}}{\phi_{1 / 2}} .
$$

Had we introduced a quadratic term in $y$ in the ansatz for the BD field in Eq. (24), we would end up with a third constraint

$$
\left[\phi^{\prime}\right]_{0}=-\left[\phi^{\prime}\right]_{1 / 2} .
$$

We must note that this constraint is formally equivalent to (27) and (28) in the sense that the three constraints require the jump in the first derivative of a given quantity across one brane to have the same absolute value but an opposite sign as the same jump across the second brane. Since we have only two quantities to fix, namely $\rho_{*}$ and $p_{*}$, this extra relation would possibly be verified only for a discrete set of values of $\omega$ and/or $\epsilon$. Therefore by discarding the quadratic term in $\phi$ we are automatically free from this problem. In any case our ansatz can always be interpreted as a power series expansion in $y$ of a more complex solution in which case it is valid for $y$ small enough. Since we are mainly interested in the cosmology of the brane at $y=0$ we are well within the domain where it is safe to consider only the lower order terms in the power series.

Although we are not going to discuss further the problem of constraints in this paper, we suggest that a much more elegant solution could possibly be found with a varying $\omega$ in the bulk. This could in principle be implemented in the context of the so called scalar-tensor theories where $\omega=\omega(\phi)$. The third constraint would then fix the values of $\omega$ at $y=1 / 2$ as a function of $\omega$ on the brane at $y=0$, therefore partially constraining the functional form of $\omega=\omega(\phi)$. Another possibility which would make the constraints unnecessary is a model with a single brane. In that case, Eq. (21) would hold without any further ado. We must point out however that the presence of a second brane is favored for several reasons (see Refs. [3.14]). As a final remark, let us note that constraints of this type are expected to appear in models with compact extra dimensions [6]. 


\section{EXACT COSMOLOGICAL SOLUTIONS}

We will now obtain some simple solutions for $a$ and $\phi$ which will allow us to discuss cosmology in our setup. For what follows we will discard the part of our ansatz in Eq. (23) (and similarly for $n$ ) which is proportional to $y^{2}$. In this context this means that we will no longer have to worry about the constraints discussed in the previous section. As before we assume the extra space like dimension is stabilized ( $b$ is constant) and choose time such that $n_{0}=1$.

From Eq. (26) and using the jump conditions Eqs. (17 19) we obtain the Friedmann equation which reads

$$
\left(\frac{\dot{a}_{0}}{a_{0}}\right)^{2}+\frac{\dot{\phi}_{0}}{\phi_{0}}\left(\frac{\dot{a}_{0}}{a_{0}}-\frac{\omega}{6} \frac{\dot{\phi}_{0}}{\phi_{0}}\right)=\frac{1}{4(3 \omega+4)^{2} \phi_{0}^{2}}\left[\frac{\omega}{6}(3 p-\rho)^{2}+\left(2+3 \omega+\omega^{2}\right) \rho^{2}-p \rho \omega-2 p^{2}\right] .
$$

Using our ansatz in the BD field equation, we obtain again Eq. (22), as we should expect. Together with the conservation equation (20), Eqs. (22) and (32) form a complete system which will allow us to study the cosmology on the brane. A careful look at the above mentioned equations suggests that once the equation of state on the brane has been specified, it is possible to obtain a power law solution to the scale factor, BD field and the matter content on the brane as a function of time.

Here we consider only the particular cases of radiation $(p=\rho / 3)$ and dust $(p=0)$ as well as the vacuum energy case $(p=-\rho)$. For a radiation dominated Universe we obtain $\phi_{0}=$ const. from Eq. (22), which also holds for the usual BD solution in four dimensions. It is noticeable that in this case the solution is exactly the same as in the case of general relativity (see Ref. [6]):

$$
a_{0} \propto \tau^{1 / 4} ; \quad \rho_{0} \propto a_{0}^{-4} ; \quad \phi_{0}=\text { const } .
$$

For the matter dominated era the solution can be obtained from Eqs. (20), 22) and (32), and is given by

$$
a_{0} \propto \tau^{\frac{3 \omega+3+\sqrt{3} \sqrt{2 \omega+3}}{9 \omega+12}} ; \quad \rho_{0} \propto a_{0}^{-3} ; \quad \phi_{0} \propto \tau^{\frac{1+\sqrt{3} \sqrt{2 \omega+3}}{3 \omega+4}},
$$

where we have taken the growing mode for the scale factor and we have restricted to large $\omega$ by truncating terms $\mathcal{O}\left(1 / \omega^{2}\right)$. It is important to notice that for large $\omega$ the solutions approach the brane general relativity case $a \propto \tau^{1 / 3}$. If the solution for the scale factor in the 4 dimensional BD case is written as $a \propto \tau^{\alpha}$ then in our model the solution takes the form $a \propto \tau^{\alpha / 2+\mathcal{O}(1 / \sqrt{\omega})}$. In our situation however the expansion during the dust era is faster compared with that in the general relativity case. This will have some implications during the rapid oscillations of the scalar field in our brane after inflation and during preheating when the expansion mimics that of a pressureless fluid.

It is important to mention here that our results are valid only prior to nucleosynthesis and at late times the Friedmann equation should converge to the standard case with $H \propto \sqrt{\rho}$ in order to satisfy the stringent constraints coming from big bang nucleosynthesis. For this to happen one may need to take the brane tension into account [6]. Denoting the brane tension by $\rho_{\Lambda}$, the total energy density in the brane will be given by $\rho_{\text {tot }}=\rho_{\Lambda}+\rho$ and the square of this quantity will include the term $2 \rho_{\Lambda} \rho$ linear in $\rho$. For $\rho_{\Lambda}>1 \mathrm{MeV}^{4}$ one can ensure compatibility with nucleosynthesis constraints. In this way for energy scales larger than the brane tension we have the non-standard behaviour, while as the universe expands and cools down we recover the standard behaviour.

Finally, we discuss the cosmology of the vacuum energy dominated stage. From the energy conservation equation we see that false vacuum inflation $(\rho=$ constant) is possible in this case for large enough values of $\omega$. The scale factor and $\mathrm{BD}$ field are given by

$$
a_{0}(\tau) \propto \tau^{\frac{3 \omega}{16}} \phi_{0}(\tau) \propto \tau .
$$

The rate of expansion during inflation in the brane is therefore slower when compared with the 4 dimensional BD case $a \propto \tau^{\omega+1 / 2}$. As for the BD field, in the 4 dimensional case $\phi \propto \tau^{2}$ and the evolution is also slower in the brane model.

Note that if the energy conservation equation (20) was not satisfied as in the models studied in 222 24] then false vacuum inflation would not be possible because due to matter leaking from the brane, even for the case $p=-\rho$ it would not possible to keep the energy density constant anymore.

An interesting possibility which we have not analyzed here is the case where the dynamics in the brane is driven by the evolution of the fifth dimension. A more detailed study of the cosmology in the model described in this paper, including an analysis of the primordial perturbation spectra produced by inflation in our setup will be discussed elsewhere. 


\section{CONCLUSIONS}

We have derived the effective four dimensional field equations for the brane cosmology in presence of the BD field in five dimensions. In our scenario, the conservation equation for the matter field stuck to the brane still holds in spite of the BD field present in the bulk. This is due to the fact that our calculation was done in the Jordan frame. Some global solutions with two branes such that the fifth dimension has an orbifold symmetry were discussed. Finally we obtained the modified Friedmann equation in the brane and cosmological solutions in the BD brane setup were discussed.

\section{ACKNOWLEDGMENTS}

L.E.M is supported by FCT (Portugal) under contract PRAXIS XXI BPD/14163/97. A. M. is supported by INLAKS foundation. The authors are thankful to Jim Lidsey for fruitful discussions. We are extremely grateful to Hassan Firouzjahi and Christophe Grojean for pointing out an error in our calculation which has led to a complete revision of our paper.

[1] V. A. Rubakov and M. E. Shaposhnikov, Phys. Lett. B 125, (1983) 136; K. Akama, "Pregeometry" in Lecture Notes in Physics, 176, Gauge Theory and Gravitation,Proceedings, Nara, 1982, (Springer-Verlag), edited by K. Kikkawa, N. Nakanishi and H. Nariai, 267-271; hep-th/0001113.

[2] P. Horava and E. Witten, Nucl. Phys. B 460 (1996) 506; Nucl. Phys. B 475 (1996) 94.

[3] A. Lukas, B. A. Ovrut, K. Stelle and D. Waldram, Phys. Rev. D 60 (1999) 086001; J. E. Ellis, Z. Lalak, S. Pokorski and W. Pokorski, Nucl. Phys. B 540(1999) 149.

[4] L. Randall and R. Sundrum, Phys. Rev. Lett. 83, (1999) 3370.

[5] A. Mazumdar and J. Wang, Phys. Lett. B 490 (2000) 251.

[6] P. Binetruy, C. Deffayet and D. Langlois, Nucl. Phys. B 565 (2000) 269; P. Binetruy, C. Deffayet, U. Ellwanger and D. langlois, Phys. Lett.B 477 (2000) 269; J. M. Cline, C. Grojean and G. Servant, Phys. Rev. Lett. 83 (1999) 4245; T. Shiromizu, K. Maeda and M. Sasaki, gr-qc/9910076.

[7] N. Kaloper, Phys. Rev. D 60 (1999) 123506; M. Cevetic and Jing Wang, Phys. Rev. D 61 (2000) 124020; R. Maartens, hepth/0004166; C. van de Bruck, M. Dorca, R. Brandenberger and A. Lukas, hep-th/0005032; D. Langlois, hep-th/0005025; B. Grinstein, D. R. Nolte and W. Skiba, hep-th/ 005001; K. Koyama and J. Soda, hep-th/ 005239; L. Anchordoqui, C. Nunez, K. Olsen, hep-th/0007064.

[8] A very incomplete list includes: T. Nihei, Phys. Lett. B 465 (1999) 81; C. Csaki, M. Graesser, C. Kolda and J. Terning, Phys. Lett. B 462 (1999) 34; P. Kanti, I. I. Kogan, K. A. Olive and M. Pospelov, Phys. Lett. B 468 (1999) 31; Phys. Rev. D 61 (2000) 106004; P. Kraus, JHEP 9912 (1999) 011; H. B. Kim and H. D. Kim, Phys. Rev. D 61 (2000) 064003; H. B. Kim, Phys. Lett. B 478 (2000) 285; H. Stoica, S. H. Tye, I. Wasserman, Phys. Lett. B 482 (2000) 205; C. Csaki, M. Graesser, L. randall and J. Terning, hep-ph/9911406; R. Maartens, D. Wands, B. A. Bassett and I. Heard, Phys. Rev. D 62 (2000) 041301; R. N. Mohapatra, A. Perez-Lorenzana and C. A. de Sousa Pires, hep-ph/0003328; L. E. Mendes and A. Liddle, astro-ph/0006020; L. Anchordoqui, K. Olsen,hep-th/0008102; E. J. Copeland, A. R. Liddle and J. Lidsey, astro-ph/0006421.

[9] A. Mazumdar, hep-ph/0007269, hep-ph/0008087.

[10] N. Arkani-Hamed, S. Dimopoulos, N. Kaloper and R. Sundrum, Phys. Lett. B 480 (2000) 193.

[11] S. Kachru, M. Schluz and E. Silverstein, hep-th/0001206.

[12] P.Binetruy, J. M. Cline and C. Grojean, hep-th/007029.

[13] S.Nojiri and S. D. Odintsov, Phys. Lett. B 484 (2000) 119; S. Nojiri, S. D. Odintsov and O. Obergon, hep-th/5127; C. Gomez, B. Janssen and P. J. Silva, JHEP 0004, (2000) 024; N. Alonso-Albereca, B. Janssen and P. J. Silva, JHEP 0004, (2000) 027.

[14] M. J. Duff, J. T. Liu and K. Stelle, hep-th/0007120.

[15] S. Weinberg, "Gravitation and cosmology: principles and applications of the general theory of relativity", John Wiley \& Sons, 1972.

[16] J. D. Barrow and J. P.Mimoso, Phys. Rev. D. 50, (1994) 3746.

[17] W. Israel, Nuovo Cimento B (1966) 44.

[18] J. E. Lidsey, D. Wands, E. J. Copeland, hep-th/9909061.

[19] W. D. Goldberger and M. B. Wise, Phys. Rev. Lett. 83 (1999) 4922. 
[20] D. La and P. J. Steinhardt, Phys. Rev. Lett. 62, (1989) 376; C. Mathiazhagen and V. B. Johri, Class. Quantum Grav. 1 (1984) L29.

[21] C. Barcelo and M. Visser, gr-qc/0008008, hep-th/0009032.

[22] K. Maeda and D. Wands, hep-th/0008188.

[23] A. Mennim and R. Battye, hep-th/0008192.

[24] C. van de Bruck, M. Dorca, C. J. A. P. Martins and M. Perry, hep-th/0009056. 